\title{
A tritrophic signal that attracts parasitoids to host-damaged plants withstands disruption by non-host herbivores
}

\author{
Matthias Erb, Nicolas Foresti, Ted CJ Turlings ${ }^{*}$
}

\begin{abstract}
Background: Volatiles emitted by herbivore-infested plants are highly attractive to parasitoids and therefore have been proposed to be part of an indirect plant defense strategy. However, this proposed function of the plantprovided signals remains controversial, and it is unclear how specific and reliable the signals are under natural conditions with simultaneous feeding by multiple herbivores. Phloem feeders in particular are assumed to interfere with plant defense responses. Therefore, we investigated how attack by the piercing-sucking cicadellid Euscelidius variegatus influences signaling by maize plants in response to the chewing herbivore Spodoptera littoralis.

Results: The parasitoid Cotesia marginiventris strongly preferred volatiles of plants infested with its host $S$. littoralis. Overall, the volatile emissions induced by $S$. littoralis and E. variegatus were similar, but higher levels of certain wound-released compounds may have allowed the wasps to specifically recognize plants infested by hosts. Expression levels of defense marker genes and further behavioral bioassays with the parasitoid showed that neither the physiological defense responses nor the attractiveness of $S$. littoralis infested plants were altered by simultaneous $E$. variegatus attack.

Conclusions: Our findings imply that plant defense responses to herbivory can be more robust than generally assumed and that ensuing volatiles convey specific information about the type of herbivore that is attacking a plant, even in complex situations with multiple herbivores. Hence, the results of this study support the notion that herbivore-induced plant volatiles may be part of a plant's indirect defense stratagem.
\end{abstract}

\section{Background}

Predators and parasitoids are important natural enemies of herbivorous insects. By reducing the abundance of herbivores, they can help protect plants from damage. Parasitoids in particular can use herbivore-induced plant volatiles (HIPVs) as host-searching cues [1,2]. Such volatile-mediated tritrophic interactions have a considerable potential to shape ecosystem dynamics [3], but it remains unclear to what extend the plant signals are emitted by the plant to specifically attract natural enemies of herbivores [4]. If HIPVs are indeed emitted by the plant to attract the third tropic level, specificity of the signals should be an important aspect of the interactions [5]. This is particularly relevant under natural conditions, where plants are often attacked by non-hosts or

\footnotetext{
* Correspondence: ted.turlings@unine.ch

Laboratory for Fundamental and Applied Research in Chemical Ecology, Institute of Biology, University of Neuchâtel, Neuchâtel, Switzerland
}

by multiple herbivores simultaneously. While specialist parasitoids can distinguish between plants attacked by hosts and plants attacked by non-hosts using HIPV cues [6], the impact of non-hosts feeding on the same plant as the host could be problematic. Of the few studies on this topic, most seem to suggest that non-hosts (or nonprey in the case of predators) may interfere with the plant's responses in several ways: They can indirectly change plant resistance [7-9] and therefore influence feeding behavior and subsequent HIPV induction of hosts, influence the induction of plant defenses on the physiological level via positive or negative cross-talk $[10,11]$ or induce volatile bouquets that either mask [12] or distort $[13,14]$ host/prey-finding cues. Yet, if HIPVs have evolved in the context of tritrophic interactions, it can be expected that in tightly co-evolved systems, parasitoids will be able to "tolerate" the effects of non-host herbivores on the plant and recognize volatiles 
indicating host presence, even in a complex situation with multiple herbivore attacking the same plants. Similarly, plants should have evolved to minimize the negative interference of herbivores with their defense responses targeted at other attackers. To date, little is known about this alleged robustness of volatile-mediated tritrophic interactions.

In the case of parasitoids, their capacity to learn to associate particular volatile blends with the presence of hosts may help them to cope with environmental complexity [15]: many parasitoids are more attracted to a specific volatile blend after exposure during host encounters [16]. This may also allow parasitoids to reduce the impact of the negative interference of nonhost herbivores on host-induced volatiles [17].

Typical examples of negative interference between herbivores come from research comparing insects with different feeding styles. Chewing herbivores predominantly activate jasmonic acid (JA) and ethylene (ET) dependent defenses $[18,19]$, whereas many piercingsucking insects appear to induce defense-pathways commonly associated with pathogens $[10,20,21]$. The silverleaf whitefly Bemisia tabaci for example induces salicylic acid (SA)-dependent defenses and suppresses jasmonic acid (JA)-dependent plant reactions in Arabidopsis thaliana [22], possibly via classical JA/SA crosstalk $[23,24]$. In a particularly illustrative recent study, it was found that $B$. tabaci reduces the attractiveness of spider mite infested lima beans to predatory mites [25]. The effects of piercing-sucking insects on plant defense are also evident at the gene expression level: In A. thaliana, B. tabaci induces the SA-responsive genes $P R 1$ and $P R 5$ and reduces the expression of the JA markers PDF1.2 and VSP [22]. In lima bean, the expression of $L O X$, a key enzyme in JA-biosynthesis is repressed by $B$. tabaci alongside PIOS, the gene coding for the enzyme $\beta$-ocimene synthase [25]. The evident potential of piercing sucking insects to interfere with the responses of plant to other herbivores makes them important factors to consider in studies on the functionality of plant defenses in multitrophic systems.

This prompted us to conduct experiments on the impact of the piercing-sucking leafhopper Euscelidius variegatus (Hemiptera : Cicadellidae; Kirschbaum 1858) on volatile emissions of maize plants and the tritrophic interaction involving maize, the lepidopteran pest Spodoptera littoralis (Boisduval) (Lepidoptera: Noctuidae) and the generalist parasitoid Cotesia marginiventris (Cresson) (Hymenoptera: Braconidae). C. marginiventris is a solitary larval endoparasitoid that can use a broad range of noctuid moths, including many maize pests, as hosts. It is generally highly responsive to induced volatile cues provided by the host-damaged plants, including maize $[16,26]$. Leafhoppers like $E$. variegatus co-occur with chewing lepidopteran larvae in maize agroecosystems as well as in nature on teosinte, the wild ancestor of maize [27], making the system a logical candidate for the current study. Based on the results from earlier studies (see above), we hypothesized that $E$. variegatus affects the plant's response to $S$. littoralis, thereby interfering with the ability of $C$. marginiventris to locate its host with the use of HIPVs. Following this, we specifically tested if $C$. marginiventris is able to distinguish HIPVs from hosts (E. variegatus) and non-host (S. littoralis) and if simultaneous $E$. variegatus attack affects the attractiveness of $S$. littoralis infested plants. The assays presented here took into account the effect of previous encounters with hosts and host-associated odours, which can strongly affect the responses of parasitoids, including C. marginiventris $[16,17]$, through associative learning [28].

\section{Methods}

\section{Insects and insect treatments}

The cicadellid E. variegatus was reared on 4-8 week old barley plants in plastic Bugdorm cages (Megaview, Taiwan) under standardized conditions $\left(25^{\circ} \mathrm{C}, 16: 8 \mathrm{~h} \mathrm{~L} /\right.$ D). The caterpillar S. littoralis (Boisduval) (Lepidoptera: Noctuidae) and the solitary endoparasitoid C. marginiventris (Cresson) (Hymenoptera: Braconidae) were reared as previously described [29]. Adult parasitoids were kept in plastic cages at a male/female ratio of approximately $1: 2$ and were provided with moist cotton wool and honey as food source. Cages were kept in incubators $\left(25^{\circ} \mathrm{C} ; 16: 8 \mathrm{~h} \mathrm{~L} / \mathrm{D}\right)$ and transferred to the bioassay laboratory $30 \mathrm{~min}$ before the experiments. Two to four day old mated naive and experienced females were tested. For details on the training setup see D'Alessandro et al., [30]. Naïve wasps did not have any previous oviposition experience and had never before been in contact with plant odours. To experience wasps, they were either placed with the host (20 L2 S. littoralis larvae) until they had oviposited 3-5 times, or with the non-host (10 adult $E$. variegatus) during 2 minutes, while they were exposed to the odor from plants infested by E. variegatus and/or S. littoralis. This resulted in 5 different experience groups (Table 1): Host contact (S. littoralis) in presence of $S$. littoralis induced volatiles (SS), E. variegatus induced volatiles (SE) and double-infestation induced volatiles (SES) as well as non-host contact (E. variegatus) in the presence of $S$. littoralis induced volatiles (ES) and E. variegatus induced volatiles (EE). As the perception of odors in the absence of herbivorous insects does not change the responsiveness of C. marginiventris [17], this treatment was not included. The different groups of wasps were kept separately in small plastic boxes and released into the olfactometer 1-3 $\mathrm{h}$ after their experience. 
Table 1 Herbivores and odor blends used to train C. marginiventris

\begin{tabular}{lll}
\hline Experience & Contact (Insect) & Odor Blend (Plant) \\
\hline Naïve & - & - \\
SS & S. littoralis (host) & S. littoralis induced \\
SE & S. littoralis (host) & E. variegatus induced \\
SES & S. littoralis (host) & E. variegatus and S. littoralis induced \\
ES & E. variegatus & S. littoralis induced \\
& (non-host) & \\
EE & E. variegatus & E. variegatus induced \\
& (non-host) &
\end{tabular}

Naive C. marginiventris females were exposed to either host or non-host insects (column 2) in the presence of different plant odour blends (column 3 ). This resulted in differently experienced wasps (column 1).

\section{Plants and odor sources}

Maize (Zea mays, var. Delprim) was sown in plastic pots (10 cm high, $4 \mathrm{~cm}$ diameter) with commercial potting soil (Ricoter Aussaaterde, Aarberg, Switzerland) and placed in a climate chamber $\left(23^{\circ} \mathrm{C}, 60 \%\right.$ r.h., $16: 8 \mathrm{~h} \mathrm{~L} / \mathrm{D}$, 50 '000 $\mathrm{lm} / \mathrm{m}^{2}$ ). Plants used for the experiments were 10-12 d old and had 2-3 fully developed leaves. The evening before the experiments, plants were transferred to glass vessels [29] and infested with second instar S. littoralis larvae (released in the whorl of the youngest leaf) or E. variegatus adults (released freely into the vessel). For the experiment involving double-infestation, maize seedlings were inoculated with $E$. variegatus adults 48 hours before the olfactometer assay and second instar S. littoralis larvae were added the evening before the experiment.

Thirty E. variegatus adults per plant were used, a density that induced a reliable response of maize seedlings without impairing their physiology: Preliminary experiments had shown that infestation with 20 hoppers/plant resulted in a relatively weak and less consistent volatile response (data not shown), whereas densities much above 30 individuals per plant led to a phenomenon called "hopper burn", where the plant's vascular architecture is so severely impaired that the plants start displaying yellow discoloration (personal observations).

For the chewing herbivore, nine S. littoralis larvae where used in the double-infestation experiment (for both the double and the single herbivore treatments), while the density was reduced to three in the single infestation experiment (comparing S. littoralis with $E$. variegatus infested plants). This lower density of $S$. littoralis was chosen because it resulted in a similar response in the maize plants as infestation by thirty $E$. variegatus (see results), allowing for a better qualitative comparison of the plant's defensive response. After infestation, the vessels with the plants and herbivores were attached to the air supply of the olfactometer and kept under laboratory conditions $\left(25^{\circ} \mathrm{C}, 50 \%\right.$ r.h., $16: 8 \mathrm{~h}$
L/D, humidified airflow $0.3 \mathrm{l} / \mathrm{min}, 8000 \mathrm{~lm} / \mathrm{m} 2$ ). Olfactometer experiments were done the following day, between 10 A.M. and 4 P.M.

\section{Olfactometer bioassays}

The different odor sources were tested for attractiveness to parasitoids in a six-arm olfactometer as described in Turlings et al., [29]. To compare the attractiveness of plants infested with one of the two herbivores, we compared 4 sources: E. variegatus infested plants, S. littoralis infested plants, uninfested plants and clean air. To only test the attractiveness of E. variegatus infested plants, they were tested against uninfested plants. In the double-infestation experiment, attraction to S. littoralis infested plants was compared to plants infested with both herbivores and uninfested plants. In every experiment, one arm per odor source was used. The remaining arms were connected to empty vessels and carried clean, humidified air only. The position of the odor sources was randomly assigned for each experimental run to avoid position-bias. Purified and humidified air entered each odor source vessel at $1.2 \mathrm{l} / \mathrm{min}$ (adjusted by a manifold with six flowmeters, Analytical Research System, Gainesville, FL, USA) via Teflon tubing and carried the volatiles through to the olfactometer compartment. Half of the air $(0.6 \mathrm{l} / \mathrm{min} /$ olfactometer arm $)$ was pulled out via volatile collection traps that were attached to a port on top of each odor source vessel (see "Collection and analyses of HIPVs"). These traps, as well as the wasp release chamber were connected to a vacuum pump via Tygon tubing and flow meters, and airflows were balanced with a pressure gauge. Wasps were released in groups of six into the central part of the olfactometer, alternating between groups of naive and experienced wasps, and after $30 \mathrm{~min}$ the wasps that had entered an arm of the olfactometer were counted and removed. Wasps that did not enter an arm after this time were removed from the central part of the olfactometer and considered as "no choice." Each experiment was performed 6-8 times on different days. This resulted in 6-8 independent replicates for each olfactometer setup.

\section{Collection and analysis of HIPVs}

HIPVs of each odor source were collected during the olfactometer bioassay on a Super-Q trap (25 mg, 80-100 mesh; Alltech Associates, Deerfield, IL, USA, described by Heath \& Manukian, [31]. Each trap was attached horizontally to the elbow of an odor source vessel and connected via Tygon tubing to a flowmeter (Analytical Research System) and a vacuum pump. Air carrying the volatiles was pulled through each trap at a rate of $0.6 \mathrm{l} / \mathrm{min}$ during each behavioral bioassay. Afterwards, the traps were extracted with $150 \mu \mathrm{l}$ dichloromethane 
(Suprasolv; Merck, Dietikon, Switzerland), and $200 \mathrm{ng}$ of n-octane and n-nonyl acetate (Sigma, Buchs, Switzerland) in $10 \mu \mathrm{l}$ dichloromethane were added to the samples as internal standards. All extracts were stored at $-76^{\circ} \mathrm{C}$ until analyses. Traps were washed with $3 \mathrm{ml}$ dichloromethane before they were reused for a next collection. HIPVs of the experiments were identified with a gas chromatograph (Agilent 6890 Series GC system G1530A) coupled to a mass spectrometer that operated in electron impact mode (Agilent 5973 Network Mass Selective Detector; transfer line $230^{\circ} \mathrm{C}$, source $230^{\circ} \mathrm{C}$, ionization potential $70 \mathrm{eV}$, scan range 33-280 amu). A 2- $\mu \mathrm{l}$ aliquot of each sample was injected in the pulsed splitless mode onto an apolar capillary column (HP-1, $30 \mathrm{~m}, 0.25 \mathrm{~mm}$ ID, $0.25 \mu \mathrm{m}$ film thickness; Alltech Associates). Helium at constant flow $(0.9 \mathrm{ml} / \mathrm{min})$ was used as carrier gas. After injection, the column temperature was maintained at $40^{\circ} \mathrm{C}$ for $3 \mathrm{~min}$ and then increased to $100^{\circ} \mathrm{C}$ at $8^{\circ} \mathrm{C} / \mathrm{min}$ and subsequently to $200^{\circ} \mathrm{C}$ at $5^{\circ} \mathrm{C} / \mathrm{min}$ followed by a postrun of $5 \mathrm{~min}$ at $250^{\circ} \mathrm{C}$. The detected volatiles were identified by comparison of their mass spectra with those of the NIST 05 library, by comparison of their spectra and retention times with those of authentic standards, and by comparison of retention times with those from previous analyses [32]. Precise quantification of the identified volatiles was carried out using an Agilent 6850 gas chromatograph with a flame ionization detector. A 3- $\mu \mathrm{l}$ aliquot of each sample was injected in pulsed splitless mode onto the same type of column as above at a constant pressure of 18.55 psi. The column temperature ramping was as described above.

\section{Analysis of gene expression}

The leaves from plants used in the olfactometer experiments were harvested and flash-frozen in liquid nitrogen. Based on the volatile profiles, 5 representative samples of each treatment were chosen and ground to a fine power under liquid nitrogen. Total RNA was then extracted using Quiagen RNA-Easy extraction kits following the manufacturer's instructions. The quality of the RNA was assessed by photometry and gel electrophoresis. To remove contaminant genomic DNA, all samples were treated with Ambion DNAse following the manufacturer's protocol. cDNA was then synthesized using Invitrogen Super-Script III reverse transcriptase according to the manufacturer's instructions. Based on current knowledge about the molecular basis of maize responses to insect attack, we used $Z m-B 73 L O X$ [33], $Z m-A O C$ and $Z m-A O S$ [34] as markers for the induction of the octadecanoid pathway, Zm-SerPIN [33], Zm$M P I$ and $Z m-B x 1$ [35] as markers for the induction of direct defenses and $Z m-H P L$ [36], Zm-TPS10 [37], ZmTPS23 [38] and Zm-IGL [39] as markers for volatile induction. Quantitative reverse transcriptase real time polymerase chain reactions (q-PCR) where carried out using the following gene-specific primers: $Z m-A O S \mathrm{~L}$ : acctgttcacgggcacctac; R:cgaggagcgaggagaagttg. $Z m-A O C$ L: cccttcaccaacaaggtgt; $\mathrm{R}$ : accgagatgtggccgtagtc. Zm-B73LOX L: gcgacaccatgaccatcaac; R: gctcggtgaagttccagctc. $Z m$-SerPIN L: gacggaggaggaaggaggag; R: acctgatgcactgcttgcac. $Z m-M P I$ L: atgagctccacggagtgc; R: acctgatgcactgcttgcac. $Z m-B X 1$ L: cccgagcacgtaaagcagat; $\mathrm{R}$ : cttcatgcccctggcatact. $Z m-H P L$ L: acttcggcttcaccatcctg; $\mathrm{R}$ : gtagtagcccggccagatga; Zm-IGL L: gcctcatagttcccgacctc; R: gaatcctcgtgaagctcgtg. Zm-TPS1O L: tgtgtccacggtccaatgtt; R: gtccgctgtccttgcaaaat. Zm-TPS23 L: tctggatgatgggagtcttctttg; R: gcgttgccttcctctgtgg. The qPCR mix consisted of 5 ul Quantace Sensimix containing Sybr Green I, 3.4 ul H20, $100 \mathrm{nmol}$ of each primer $(2 \times 0.3 \mathrm{ul} \mathrm{H} 20)$ and $1 \mathrm{ul}$ of cDNA sample. Q-PCR was carried out using 45 cycles with the following temperature curve: $10 \mathrm{~s} 95^{\circ} \mathrm{C}, 20 \mathrm{~s} 60^{\circ}, 15 \mathrm{~s} 72^{\circ}$. The final melt curve was obtained by ramping from 68 to $98^{\circ} \mathrm{C}$ in $1^{\circ} \mathrm{C}$ steps every $5 \mathrm{~s}$. To determine primer efficiencies and optimal quantification thresholds, a dilution series of a cDNA mix consisting of $4 \mathrm{ul}$ solution from every sample was created. Six 10-fold dilution steps were carried out to determine primer efficiencies and the standard curve was included into every q-PCR run. The final obtained $\mathrm{Ct}$ values (using the automated threshold determination feature of the Rotor-Gene 6000 software) were corrected for the housekeeping gene $\mathrm{GapC}$ and normalized to control levels to obtain average fold changes of treated plants.

\section{Herbivore performance}

To test for a possible effect of $E$. variegatus infestation on the growth and feeding activity of $S$. littoralis, the double-infestation setup (see above) was used. Nine second instar S. littoralis larvae were weighed before the experiment and were then put on maize seedlings that had either been infested with E. variegatus or had initially been left herbivore free. After the olfactometer tests ( $20 \mathrm{~h}$, see above), the larvae were removed from the plants and weighed again to determine their change in body mass. This procedure was carried out for 5 olfactometer runs (see above), resulting in 5 replicate values for caterpillar growth per treatment. Our previous studies on this system have shown that resistance against S. littoralis can reliably be quantified with feedingbioassays ranging from $6-24 \mathrm{~h}[8,33,40]$.

\section{Statistical analysis}

The relationship between parasitoids' behavioral responses and the different odor sources offered in the six-arm olfactometer was examined with a log-linear model (a generalized linear model, GLM). As the data 
did not conform to simple variance assumptions implied in using the multinomial distribution, we used quasilikelihood functions to compensate for the overdispersion of wasps within the olfactometer [29]. The model was fitted by maximum quasi-likelihood estimation in the software package $\mathrm{R}$ ( $\mathrm{R}$ : A language and Environment for Statistical Computing, Version 1.9.1, Vienna, Austria, 2006, ISBN 3-900051-07-0 http://www.R-project.org), and its adequacy was assessed through likelihood ratio statistics and examination of residuals. The amounts of volatiles and gene expression data were analyzed by using ANOVAs followed by Holm-Sidak post-hoc tests. Datasets that were not normally distributed were transformed prior to analysis. Where transformation did not resolve non-normality or unequal variances, ANOVA's on ranks followed by Dunn's or Student-Newman-Keul's post-hoc tests were used.

\section{Results}

\section{Parasitoid attraction}

To test whether the parasitoid C. marginiventris is able to distinguish between volatile blends from plants infested with its host $S$. littoralis and volatiles emitted by plants infested with the non-host $E$. variegatus, we performed an olfactometer assay giving the wasps a choice between uninfested, S. littoralis-, and E. variegatus-infested plants. Irrespective of their previous experience, C. marginiventris exhibited a strong preference for odors from host-infested plants (Figure 1a and 1b). E. variegatus-infested plants were even less attractive than control plants. Interestingly, previous contact with $E$. variegatus over a period of 2 minutes drastically reduced the overall responsiveness of the wasps and the choice for the odor of host-infested plants: Compared to naïve and host-experienced wasps, wasp choice was reduced by $50 \%$ after they had contacted $E$. variegatus while being exposed to the odor of E. variegatus infested plants and by two thirds after they encountered $E$. variegatus in association with the odor of S. littoralis-infested plants (Figure 1b). This apparent effect of negative association was reflected in a significant effect of the type of experience and the interaction term (treatment"experience) in the GLM. Because C. marginiventris seemed to prefer control plants over $E$. variegatus-infested plants in the first olfactometer assay, we tested if the E. variegatus infestation had a repellent effect on the parasitoid. When offered an uninfested plant and an E. variegatus infested plant only, C. marginiventris showed a preference for odors from E. variegatus-infested plants (Figure $2 a)$, irrespective of previous experience (Figure 2b). To assess whether E. variegatus attack affected the plants' response to $S$. littoralis, we infested the plants with $E$. variegatus and $S$. littoralis simultaneously or with $S$. littoralis only. Overall, C. marginiventris was equally attracted to double infested plants compared to plants infested with $S$. littoralis only (Figure 3a). However, parasitoids with previous positive experience, while perceiving the odor of double infested plants, shifted their preference in favor of this odor (Figure 3b). S. littoralis gained similar amounts of weight irrespective of the presence of E. variegatus (Figure 4), indicating similar feeding activity.

\section{Volatile profiles}

The volatile profiles analyzed from the olfactometer experiments described above show that plants infested with 30 E. variegatus adults emitted similar amounts of terpenoids as seedlings infested with $3 \mathrm{~S}$. littoralis caterpillars (Figure 1c). Therefore, the overall induction resulting from the two chosen herbivore densities was comparable. Unlike $S$. littoralis, E. variegatus feeding did not result in detectable release of the green leaf volatiles (GLVs) (Z)-3-hexanal and (E)-2 hexenal, whereas the emission of the other two GLVs, (Z)-3hexen-1-ol and (E)-2-hexenyl acetate was not significantly different between the two herbivore treatments. Of the other compounds, only indole was emitted in lower amounts by E. variegatus-infested plants compared to S. littoralis-infested plants (Figure 1c). The analyzed volatile profiles of the second olfactometer experiment ( $E$. variegatus vs. control) confirmed that E. variegatus induces the same 18 compounds commonly found in S. littoralis-infested plants, apart from the two GLVs $(Z)$-3-hexanal and $(E)$-2-hexenal (Figure 2c). The volatile compounds induced by simultaneous E. variegatus and S. littoralis attack were qualitatively and quantitatively similar to the profile induced by $S$. littoralis only (Figure 3c). Compounds that were not identified by comparing retention times and spectra with those of pure standards are indicated in Figures 1 and 2 with superscript $\mathrm{N}$, and their identity should be considered tentative.

\section{Gene expression profiles}

To gain insight into the genetic basis for the observed volatile responses, we profiled gene expression patterns of plants infested by E. variegatus, S. littoralis and both herbivores simultaneously. The putative octadecanoid biosynthesis genes were induced by both $E$. variegatus and $S$. littoralis, with $Z m-A O S$ showing a reaction to $S$. littoralis only and $Z m-A O C$ being more strongly induced by the caterpillar (Figure 1d). The markers for direct defenses, $Z m$-SerPIN, Zm-MPI, two proteinase inhibitor genes, and $Z m-B X 1$, a key gene for the synthesis of hydroxamic acids, also responded to both herbivores, with $Z m$-SerPIN showing a stronger reaction to $S$. littoralis. Three genes implicated in volatile production $(Z m-I G L$, the indole synthase, $Z m-T P S 10$ and $Z m$ - 

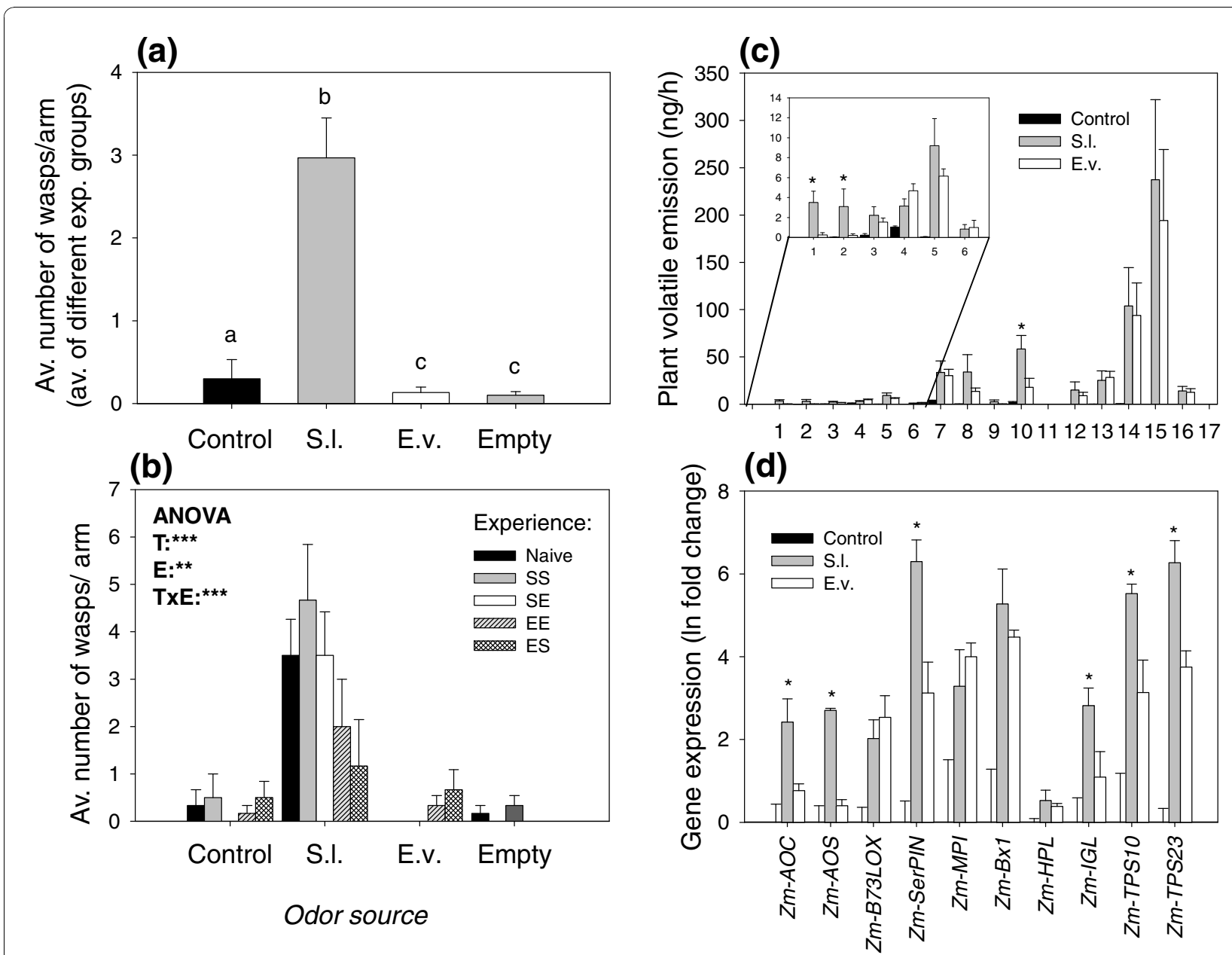

Figure 1 Influence of E. variegatus and S. littoralis infestation on parasitoid attraction, volatile emission and defense gene expression. (a): Choice of C. marginiventris, shown as the average numbers (+SE) of wasps per release (groups of 6) and olfactometer arm. Different experience types are pooled. Control $=$ Uninfested plant; S.I. = S. littoralis infested plant; E.v. $=$ E. variegatus infested plant; Empty $=$ Empty arms. Different letters indicate significant differences between treatments $(p<0.05)(n=6)$. (b): Average choice of $C$. marginiventris parasitoids with different previous host- or non-host experience. Naive $=$ No experience; $S S=$ Host-presence with $S$. littoralis induced plant odours; SE = Host presence with $E$. variegatus induced plant odours. $\mathrm{EE}=$ Non-host presence with $E$. variegatus induced plant odours; $\mathrm{ES}=$ Non-host presence with S. littoralis induced odours. Stars denote significant effects of treatment (T), experience (E) and the interaction (TxE) $\left({ }^{*} p<0.05,{ }^{* *} p<0.01,{ }^{* * *} p<\right.$ 0.001). (c): Average volatile emission (+SE) of herbivore infested maize seedlings. $1=$ (Z)-3-hexenal; $2=(\mathrm{E})$-2-hexenal; $3=(\mathrm{Z})$ - 3 -hexen-1-ol; $4=\beta$ myrcene; 5 = (Z)-3-hexenyl acetate; $6=$ (Z)- $\beta$-ocimene ${ }^{N} ; 7=$ linalool; $8=(3 \mathrm{E})$-4,8-dimethyl-1,3,7-nonatriene (DMNT); $9=$ phenethyl acetate; $10=$ indole; $11=$ methyl anthranilate; $12=$ geranyl acetate; $13=\mathrm{E}$ - $\beta$-caryophyllene; $14=(\mathrm{E})$ - $\alpha$-bergamotene; $15=\mathrm{E}-\beta$-farnesene; $16=\beta$ sesquiphellandrene $\mathrm{N}^{\mathrm{N}} 17=$ unknown sesquiterpene ${ }^{\mathrm{N}}$. Compounds denoted with " $\mathrm{N}$ " were only tentatively identified. Stars denote significant differences between $S$. littoralis and E. variegatus induced plants $(p<0.05)$. (d): Average change in gene expression (+SE) of herbivore infested maize seedlings relative to uninfested control plants. Stars in graphs (c) and (d) denote significant differences between $S$. littoralis and $E$. variegatus induced plants $(p<0.05)$.

TPS23, two major terpenoid synthases) showed a similar pattern (Figure 1d). Zm-HPL, a gene typically involved in GLV synthesis, was only significantly induced by $S$. littoralis, but there was no significant difference compared to the transcriptional activity upon E. variegatus attack. Plants attacked simultaneously by both $E$. variegatus and S. littoralis showed comparable levels of expression for all genes under investigation (Figure 3d), with the exception of $Z m-A O S$, which showed a more pronounced response upon double attack compared to S. littoralis infestation only.

\section{Discussion}

Differences in induced volatile and defense gene profiles Surprisingly, E. variegatus induced volatile profiles resembled the ones induced by $S$. littoralis in many aspects. Both herbivores induced a variety of monohomo- and sesquiterpenes, the shikimic acid pathway 

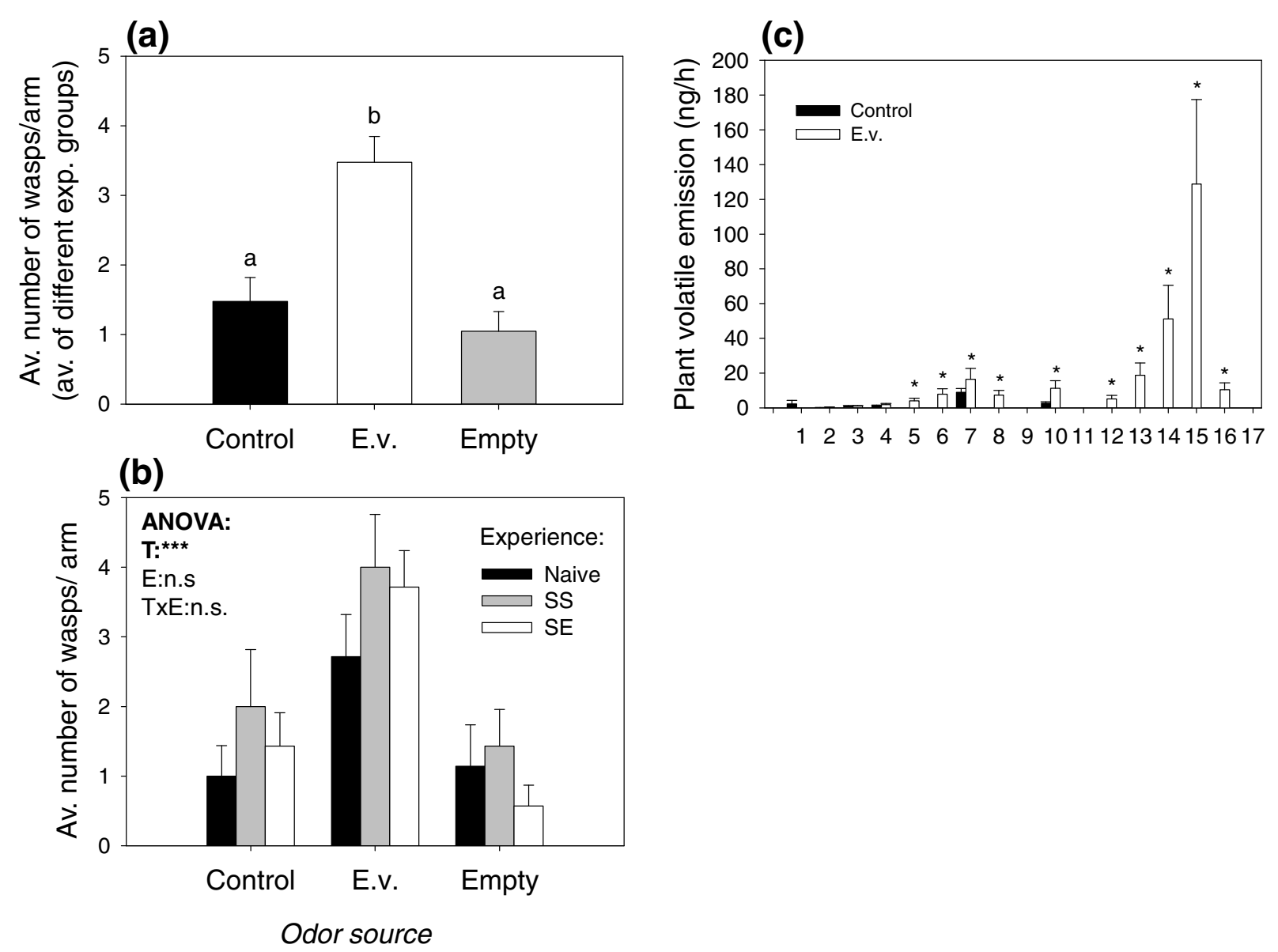

Figure 2 Influence of E. variegatus on parasitoid attraction and plant volatile emission. (a): Choice of C. marginiventris, shown as the average numbers (+SE) of wasps per release (groups of 6) and olfactometer arm. Control = Uninfested plant; E.v. = E. variegatus infested plant; Empty $=$ Empty arms. Different letters indicate significant differences between treatments $(p<0.05)$. (b): Average choice of $C$. marginiventris parasitoids with different previous host-experience. Naive = No experience; $\mathrm{SS}=$ Host-presence with $\mathrm{S}$. littoralis induced plant odours; $\mathrm{SE}=\mathrm{Host}$ presence with $E$. variegatus induced plant odours. Stars denote significant effects of treatment (T), experience (E) and the interaction (TxE) $\left({ }^{*} p<\right.$ $0.05,{ }^{* *} p<0.01,{ }^{* * *} p<0.001$ ). (c): Average volatile emission (+SE) of E. variegatus infested maize seedlings. For compound descriptions, see legend in Figure 1. Stars denote significant differences between E. variegatus induced and uninfested plants $(p<0.05)$. $(n=7)$

derived indole, as well as volatile metabolites from the oxlipin cascade, $(Z)$-3-hexen-1-ol and $(E)$-2-hexenyl acetate (Figures $1 \mathrm{c}$ and $2 \mathrm{c}$ ). This suggests that the plant's volatile response to the two herbivores is not fundamentally different, although the induction by $E$. variegatus was much weaker on a per capita basis. Sesquiterpene emissions in maize are strongly correlated with induction of JA, resulting from activation of the octadecanoid pathway [41]. Furthermore, the transcriptional profiling shows an induction of genes involved in JA biosynthesis ( $Z m-B 73 L O X$ and $Z m-A O C$, Figure $1 \mathrm{~d}$ ) by $E$. variegatus. Our results therefore imply that $E$. variegatus induces JA-dependent volatile production rather than suppress this type of defense, as has been shown for other piercing-sucking insects $[20,22]$.
The fact that $E$. variegatus did not measurably alter $S$. littoralis-induced volatile emissions (Figure 3c) and did not reduce the induced resistance the caterpillars encountered in the leaves (Figure 4) is further evidence for the absence of negative cross-talk between defense pathways in this system, as it has been reported in other cases $[22,25]$. This notion is confirmed by the transcriptional data, showing similar induction of most defenserelated genes upon S. littoralis attack irrespective of the presence of E. variegatus (Figure 3d). Only the expression of $Z m-A O S$, a putative maize allene oxide synthase involved in OPDA synthesis [34], was slightly higher in the double treatment than after caterpillar attack alone. It remains to be investigated if this higher expression leads to any changes in octadecanoid pathway dynamics. 


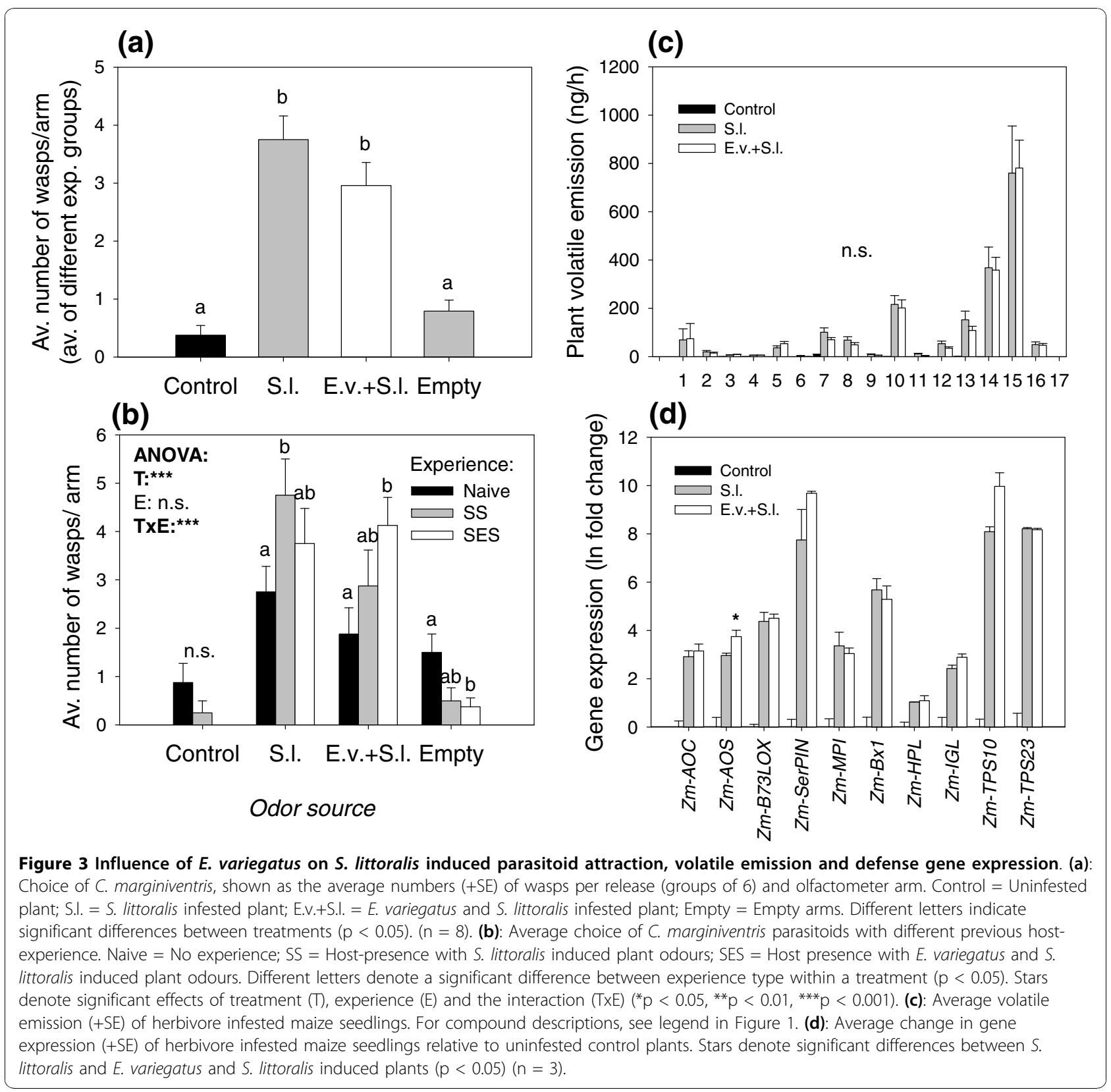

\section{Specific attraction of C. marginiventris}

C. marginiventris showed a clear preference for S. littoralis-induced blend of volatiles (Figure 1a), suggesting that the parasitoid can readily distinguish between the odors induced by the two herbivores. Both the main sesquiterpenes and aromatic compounds emitted by insectdamaged maize plants are known to be of minor importance for innate attraction of $C$. marginiventris females $[30,32,37]$, while fresh damage has been shown to be highly attractive [42]. These types of volatiles were clearly more prominent in S. littoralis-infested plants, which exhibited physical tissue damage and consequently released more $(Z)$-3-hexanal and $(E)$-2-hexenal
(Figure 1c). We thus provide evidence for the notion that the key attractants for this parasitoid are likely to be found in the blend directly released from wounded sites. The fact that $C$. marginiventris was attracted to $E$. variegatus infested plants in the absence of host-induced volatiles (Figure 2a) demonstrates that there is no repellent effect of $E$. variegatus induced volatiles per se. This could mean that the key attractants responsible for the attractiveness of the $S$. littoralis-induced blend were also emitted by $E$. variegatus-infested plants, albeit in much lower quantities, prompting the insect to respond to them only in the absence of stronger cues. Alternatively, C. marginiventris may simply choose to follow "the 


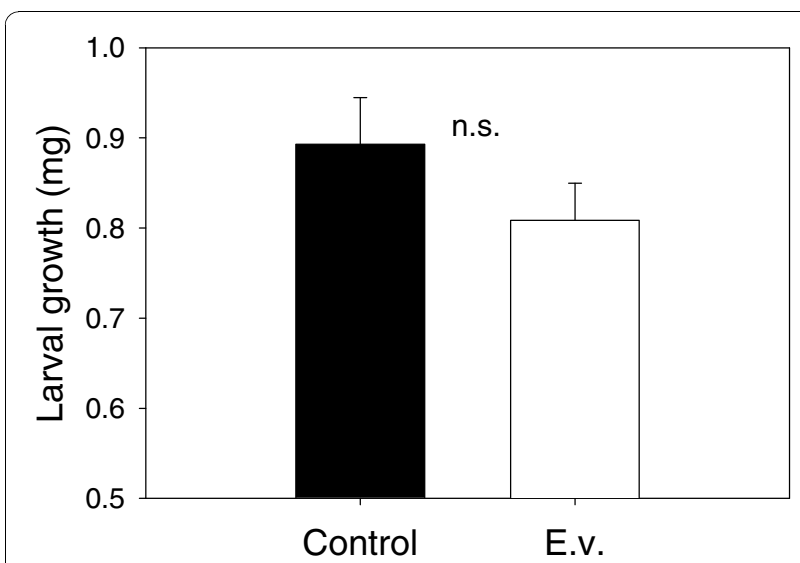

Figure 4 Influence of $E$. variegatus on maize resistance to $S$. littoralis. Average S. littoralis growth (+SE) over 18 hours on uninfested (Control) and E. variegatus infested (E.v.) plants $(n=5)$.

most promising trail" that is present in an environment by using secondary cues in the absence of primary attractants. This behavioral plasticity could be especially important for generalist parasitoids, as they have to be able to exploit a broad range of host-induced cues [28].

The attractiveness of $S$. littoralis-infested plants was not reduced when $E$. variegatus was present on the same plants (Figure 3a), indicating a robust host-finding behavior of the parasitoid. This contrasts with other studies documenting effects of multiple herbivory on tritrophic systems. Rodriguez-Sanoa et al. [43] found that C. marginiventris was more attracted to tomato plants infested with both the aphid Macrosiphum euphorbiae and the caterpillar host Spodoptera exigua than plants infested with $S$. exigua only. Similarly, the green peach aphid Myzus persicae increased the attractiveness of spider-mite induced pepper plants to a generalist predator [44]. Zhang et al. [25] on the other hand show that $B$. tabaci infestation reduces the attractiveness of spider mite induced plant to predatory mites. As shown in the same study, the interference of phloem feeders with host plant physiology can be density dependent, and it is possible that this is one of the reasons for the varying results in the literature. In maize agroecosystems, as well as in populations of teosinte, the wild ancestor of maize in Mexico, leafhoppers densities do not exceed 15 individuals per plant $[45,46]$. Early instar Spodoptera spp. larvae can initially occur at densities of up to 100 individuals per plant (G. von Merey, unpublished). Thus, the herbivore densities used in our experiment (30 E. variegatus adults, 3- 9 S. littoralis larvae) were strongly biased to maximize the chance of finding a possible effect of the leafhoppers on the volatile signals. The fact that even under these conditions, the tritrophic interaction between maize, S. littoralis and C. marginiventris remained fully functional is strong evidence for its robustness. The $C$. marginiventris strain that was used in this study originates from Mexico, where there is a long co-evolutionary history of the parasitoid, Spodoptera spp. and the ancestors of maize. This may be another explanation for the observed robustness of the system, which is unlikely to be found in the more artificial combinations of plants and insects that have been studied in this context.

It will be interesting to assess if $C$. marginiventris is also able to distinguish host-induced volatile blends from those induced by chewing non-host herbivores. In maize, early instars of non-host stem-borers like Ostrinia numbilalis and Diatrea spp. inflict visually similar leaf-damage as Spodoptera spp., and further studies should determine if $C$. marginiventris has adapted to avoid possible mismatches at this level as well. As yet, no other study on the subject matter has taken into account the learning ability of parasitoids and predators, which can be, as shown here, an important factor affecting their foraging behavior.

\section{Associative learning by the parasitoid}

Parasitoids commonly show an ability to learn to associate specific olfactory or visual cues with the presence of hosts and food [47-50]. This associative learning is thought to help the parasitoids to optimize their foraging success by focusing on the most rewarding cues $[2,28]$. The results obtained here confirm this notion and moreover represent one of the rare examples of negative learning, whereby non-rewarding cues become less attractive [51-53]. C. marginiventris markedly decreased its response to induced maize volatiles after having perceived them during contact with $E$. variegatus adults for two minutes (Figure 1b). While such effects have been demonstrated for host-free habitats [51,52] and in the context of previously learned responses [53], we show here -to our knowledge for the first time- that the presence of non-hosts can have direct negative effects on the responsiveness of previously inexperienced parasitoids. Similar assays have shown that the responsiveness of $C$. marginiventris is not affected when it perceives odors in the absence of herbivores [17], suggesting that it is indeed the presence of a non-host rather than the absence of hosts that led to the observed reduction in choice fidelity. It is tempting to speculate that $C$. marginiventris may have adapted to specifically recognize non-hosts like leafhoppers, which it regularly encounters in nature [27].

The attractiveness of S. littoralis damaged plants was not reduced by positive experience with alternative volatile blends (Figure 1b), implying that there is a strong innate preference of $C$. marginiventris for the compounds resulting from fresh damage. This could be adaptive, as it enables the parasitoid to discriminate 
between plants attacked by chewing herbivores (potential hosts) and insects with other feeding modes (nonhosts). On the other hand, positive associative learning enabled the parasitoid to distinguish double- from single-infested plants after oviposition-experience in presence of the respective blends (Figure $3 \mathrm{~b}$ ). Rasmann and Turlings [54] found something similar when they tested the attraction of $C$. marginiventris to maize plants that were simultaneously attacked by $S$. littoralis and a belowground herbivore. In both cases the learned behavior cannot be explained by the measurable volatile profiles, as they did not differ between the two treatments (Figure 3c; [54]). This confirms the importance of minor, undetected compounds, which can be affected by the presence of the additional herbivore and that can be learned and thus affect the wasps responses. As concluded in earlier studies [32,54], future research will have to focus on these elusive signals in order to unravel the functional complexity of herbivore-induced volatiles in detail.

\section{Conclusions}

Our data show that plant-mediated signaling in the tritrophic system comprising maize, the lepidopteran pest S. littoralis, and the parasitoid C. marginiventris is not disrupted by a non-host phloem feeder. This demonstrates that this specific interaction, unlike some others, is robust and that the attraction of natural enemies of herbivores to plant signals can also function when plants are attacked by multiple antagonists. Yet, flexibility in the use of reliable cues is maintained through associative learning, which may help parasitoids to specifically focus on the odor of plants that carry potential hosts and avoid plants that are only attacked by non-hosts. These results support the still controversial notion that HIPVs, at least in part, serve as functional signals to attract the enemies of the enemies of plants $[1,3,5,55]$.

\section{Acknowledgements}

We thank Roland Reist and Syngenta (Stein, $\mathrm{CH}$ ) for the weekly shipment of the S. littoralis eggs. Xavier Pons and Belén Lumbierres Bardaji (Universitat de Lleida, ES) kindly provided E. variegatus individuals. Matthias Held helped with the statistical analysis. Research activities by ME, NF and TCJT were supported by the Swiss National Science Foundation (3100A0-122132).

\section{Authors' contributions}

ME designed the experiments, analyzed the data and wrote the manuscript. NF contributed to the experimental design, carried out the experiments and analyzed data. TCJT conceived of the study, participated in its design and helped to draft the manuscript. All authors have read and approved the final manuscript.

Received: 26 March 2010 Accepted: 15 November 2010 Published: 15 November 2010

\section{References}

1. Vet LEM, Dicke M: Ecology of infochemical use by natural enemies in a tritrophic context. Annual Review of Entomology 1992, 37:141-172.
2. Turlings TCJ, Wäckers FL: Recruitment of predators and parasitoids by herbivore-damaged plants. In Advances in Insect Chemical Ecologye. Edited by: Carde RT, Millar J. Cambridge: Cambridge University Press; 2004:21-75.

3. Kessler A, Baldwin IT: Defensive function of herbivore-induced plant volatile emissions in nature. Science 2001, 291(5511):2141-2144.

4. Allison JD, Hare JD: Learned and naive natural enemy responses and the interpretation of volatile organic compounds as cues or signals. New Phytologist 2009, 184(4):768-782.

5. Dicke M, van Loon JJA, Soler R: Chemical complexity of volatiles from plants induced by multiple attack. Nat Chem Biol 2009, 5(5):317-324.

6. De Moraes CM, Lewis WJ, Pare PW, Alborn HT, Tumlinson JH: Herbivoreinfested plants selectively attract parasitoids. Nature 1998, 393(6685):570-573.

7. Poelman EH, Broekgaarden C, Van Loon JJA, Dicke M: Early season herbivore differentially affects plant defence responses to subsequently colonizing herbivores and their abundance in the field. Molecular Ecology 2008, 17(14):3352-3365.

8. Erb M, Flors $V$, Karlen D, de Lange E, Planchamp C, D'Alessandro M, Turlings TCJ, Ton J: Signal signature of aboveground-induced resistance upon belowground herbivory in maize. Plant Journal 2009, 59(2):292-302

9. Erb M, Ton J, Degenhardt J, Turlings TCJ: Interactions between arthropodinduced aboveground and belowground defenses in plants. Plant Physiology 2008, 146(3):867-874

10. Walling LL: The myriad plant responses to herbivores. Journal of Plant Growth Regulation 2000, 19(2):195-216.

11. de Boer JG, Hordijk CA, Posthumus MA, Dicke M: Prey and non-prey arthropods sharing a host plant: Effects on induced volatile emission and predator attraction. Journal of Chemical Ecology 2008, 34(3):281-290.

12. Schroeder $R$, Hilker $M$ : The relevance of background odor in resource location by insects: A behavioral approach. Bioscience 2008, 58(4):308-316.

13. Soler R, Harvey JA, Bezemer TM: Foraging efficiency of a parasitoid of a leaf herbivore is influenced by root herbivory on neighbouring plants. Functional Ecology 2007, 21(5):969-974.

14. Shiojiri K, Takabayashi J, Yano S, Takafuji A: Infochemically mediated tritrophic interaction webs on cabbage plants. Population Ecology 2001, 43(1):23-29.

15. Turlings TCJ, Wackers FL, Vet LEM, Lewis WJ, Tumlinson JH: Learning of host-finding cues by hymenopterous parasitoids. In Insect Learning: Ecological and Evolutionary Perspectives. Edited by: Papaj DR, Lewis A. New York: Champan 1993:51-78.

16. Tamo C, Ricard I, Held M, Davison AC, Turlings TCJ: A comparison of naive and conditioned responses of three generalist endoparasitoids of lepidopteran larvae to host-induced plant odours. Animal Biology 2006, 56(2):205-220.

17. Costa A, Ricard I, Davison AC, Turlings TCJ: Effects of rewarding and unrewarding experiences on the response to host-induced odors in the generalist parasitoid Cotesia marginiventris (Hymenoptera: Braconidae). Journal of Insect Behavior 2010, 23(4):303-318.

18. Odonnell PJ, Calvert C, Atzorn R, Wasternack C, Leyser HMO, Bowles DJ: Ethylene as a signal mediating the wound response of tomato plants. Science 1996, 274(5294):1914-1917.

19. Farmer EE, Almeras $E$, Krishnamurthy V: Jasmonates and related oxylipins in plant responses to pathogenesis and herbivory. Current Opinion in Plant Biology 2003, 6(4):372-378.

20. Thompson GA, Goggin FL: Transcriptomics and functional genomics of plant defence induction by phloem-feeding insects. Journal of Experimental Botany 2006, 57(4):755-766.

21. Zhou GX, Qi JF, Ren N, Cheng JA, Erb M, Mao BZ, Lou YG: Silencing OsHILOX makes rice more susceptible to chewing herbivores, but enhances resistance to a phloem feeder. Plant Journal 2009, 60(4):638-648.

22. Zarate SI, Kempema LA, Walling LL: Silverleaf whitefly induces salicylic acid defenses and suppresses effectual jasmonic acid defenses. Plant Physiology 2007, 143(2):866-875.

23. Beckers GJM, Spoel SH: Fine-tuning plant defence signalling: Salicylate versus jasmonate. Plant Biology 2006, 8(1):1-10.

24. Pieterse CM, Van Loon L: NPR1: the spider in the web of induced resistance signaling pathways. Current Opinion in Plant Biology 2004, 7(4):456-464

25. Zhang PJ, Zheng SJ, van Loon JJA, Boland W, David A, Mumm R, Dicke M: Whiteflies interfere with indirect plant defense against spider mites in 
Lima bean. Proceedings of the National Academy of Sciences of the United States of America 2009, 106(50):21202-21207.

26. Turlings TCJ, Tumlinson JH, Lewis WJ: Exploitation of herbivore-induced plant odors by host-seeking parasitic wasps. Science 1990, 250(4985):1251-1253.

27. Moya-Raygoza G, Larsen KJ, Rauk A: Geographic and seasonal variation in size and color of adult corn leafhoppers (Hemiptera : Cicadellidae) from Mexico. Environmental Entomology 2005, 34(6):1388-1394.

28. Vet LEM, Lewis WJ, Carde RT: Parasitoid foraging and learning. In Chemical Ecology of Insects 2. Edited by: CR T, Bell WJ. New York: Chapman 1995:65-101.

29. Turlings TCJ, Davison AC, Tamo C: A six-arm olfactometer permitting simultaneous observation of insect attraction and odour trapping. Physiological Entomology 2004, 29(1):45-55.

30. D'Alessandro M, Held M, Triponez Y, Turlings TCJ: The role of indole and other shikimic acid derived maize volatiles in the attraction of two parasitic wasps. Journal of Chemical Ecology 2006, 32(12):2733-2748.

31. Heath RR, Manukian A: Development and evaluation of systems to collect volatile semiochemicals from insects and plants using a charcoal-infused medium for air purification. Journal of Chemical Ecology 1992. 18(7):1209-1226.

32. D'Alessandro M, Turlings TCJ: In situ modification of herbivore-induced plant odors: A novel approach to study the attractiveness of volatile organic compounds to parasitic wasps. Chem Senses 2005, 30(9):739-753.

33. Ton J, D'Alessandro M, Jourdie V, Jakab G, Karlen D, Held M, Mauch-Mani B, Turlings TCJ: Priming by airborne signals boosts direct and indirect resistance in maize. Plant Journal 2007, 49(1):16-26.

34. Gao XQ, Starr J, Gobel C, Engelberth J, Feussner I, Tumlinson J, Kolomiets M: Maize 9-lipoxygenase ZmLOX3 controls development, rootspecific expression of defense genes, and resistance to root-knot nematodes. Molecular Plant-Microbe Interactions 2008, 21(1):98-109.

35. Frey M, Chomet P, Glawischnig E, Stettner C, Grun S, Winklmair A, Eisenreich W, Bacher A, Meeley RB, Briggs SP, et al: Analysis of a chemical plant defense mechanism in grasses. Science 1997, 277(5326):696-699.

36. Farag MA, Fokar M, Zhang HA, Allen RD, Pare PW: (Z)-3-Hexenol induces defense genes and downstream metabolites in maize. Planta 2005, 220(6):900-909.

37. Schnee C, Köllner TG, Held M, Turlings TCJ, Gershenzon J, Degenhardt J: The products of a single maize sesquiterpene synthase form a volatile defense signal that attracts natural enemies of maize herbivores. Proceedings of the National Academy of Sciences of the United States of America 2006, 103(4):1129-1134.

38. Köllner TG, Held M, Lenk C, Hiltpold I, Turlings TCJ, Gershenzon J, Degenhardt J: A maize (E)-beta-caryophyllene synthase implicated in indirect defense responses against herbivores is not expressed in most American maize varieties. Plant Cell 2008, 20(2):482-494.

39. Frey M, Stettner C, Pare PW, Schmelz EA, Tumlinson JH, Gierl A: An herbivore elicitor activates the gene for indole emission in maize. Proceedings of the National Academy of Sciences of the United States of America 2000, 97(26):14801-14806.

40. Erb M, Köllner TG, Degenhardt J, Zwahlen C, Hibbard BE, Turlings TCJ: The role of abscisic acid and water stress in root herbivore-induced leaf resistance. New Phytologist 2010, Published online 14 Sep 2010.

41. Schmelz EA, Alborn HT, Tumlinson JH: Synergistic interactions between volicitin, jasmonic acid and ethylene mediate insect-induced volatile emission in Zea mays. Physiologia Plantarum 2003, 117(3):403-412.

42. Hoballah ME, Turlings TCJ: The role of fresh versus old leaf damage in the attraction of parasitic wasps to herbivore-induced maize volatiles. Journal of Chemical Ecology 2005, 31(9):2003-2018.

43. Rodriguez-Saona C, Chalmers JA, Raj S, Thaler JS: Induced plant responses to multiple damagers: differential effects on an herbivore and its parasitoid. Oecologia 2005, 143(4):566-577.

44. Moayeri HRS, Ashouri A, Poll L, Enkegaard A: Olfactory response of a predatory mirid to herbivore induced plant volatiles: multiple herbivory vs. single herbivory. Journal of Applied Entomology 2007, 131(5):326-332.

45. Power AG: Plant community diversity, herbivore movement, and an insect-transmitted disease of maize. Ecology 1987, 68(6):1658-1669.

46. Pons X, Lumbierres B, Lopez C, Albajes R: Abundance of non-target pests in transgenic Bt-maize: A farm scale study. European Journal of Entomology 2005, 102(1):73-79.
47. Lewis WJ, Takasu K: Use of learned odours by a parasitic wasp in accordance with host and food needs. Nature 1990, 348(6302):635-636.

48. Vet LEM, Groenewold AW: Semiochemicals and learning in parasitoids. Journal of Chemical Ecology 1990, 16(11):3119-3135.

49. Turlings TCJ, Scheepmaker JWA, Vet LEM, Tumlinson JH, Lewis WJ: How contact foraging experiences affect preferences for host-related odors in the larval parasitoid Cotesia marginiventris (Cresson) (Hymenoptera, Braconidae). Journal of Chemical Ecology 1990, 16(5):1577-1589.

50. Wackers FL, Lewis WJ: Olfactory and visual learning and their combined influence on host site location by the parasitoid Microplitis croceipes (Cresson). Biological Control 1994, 4(2):105-112.

51. Papaj DR, Snellen H, Swaans K, Vet LEM: Unrewarding experiences and their effect on foraging in the parasitic wasp Leptopilina heterotoma (Hymenoptera, Eucoilidae). Journal of Insect Behavior 1994, 7(4):465-481.

52. Takasu K, Lewis WJ: The role of learning in adult food location by the larval parasitoid, Microplitis croceipes (Hymenoptera: Braconidae). Journal of Insect Behavior 1996, 9(2):265-281.

53. Takasu K, Lewis WJ: Learning of host searching cues by the larval parasitoid Microplitis croceipes. Entomologia Experimentalis Et Applicata 2003, 108(2):77-86.

54. Rasmann S, Turlings TCJ: Simultaneous feeding by aboveground and belowground herbivores attenuates plant-mediated attraction of their respective natural enemies. Ecology Letters 2007, 10(10):926-936.

55. Heil M: Indirect defence via tritrophic interactions. New Phytologist 2008, 178(1):41-61.

doi:10.1186/1471-2229-10-247

Cite this article as: Erb et al:: A tritrophic signal that attracts parasitoids to host-damaged plants withstands disruption by non-host herbivores. BMC Plant Biology 2010 10:247.

\section{Submit your next manuscript to BioMed Central and take full advantage of:}

- Convenient online submission

- Thorough peer review

- No space constraints or color figure charges

- Immediate publication on acceptance

- Inclusion in PubMed, CAS, Scopus and Google Scholar

- Research which is freely available for redistribution

Submit your manuscript at www.biomedcentral.com/submit
C Biomed Central 